\title{
Sake Brewing and Bacteria Inhabiting Sake Breweries
}

\author{
Hiromi Nishida* \\ Department of Biotechnology, Toyama Prefectural University, Imizu, Japan
}

Keywords: bacterial flora, koji, kuratsuki, Hatsuzoe, moto, Kocuria sp., sake brewing

\section{INTRODUCTION}

Sake is a Japanese traditional fermented alcoholic drink. It is brewed using koji mold to convert the starch in rice into sugar, which is then converted into ethanol by sake yeast. Two eukaryotic microorganisms, Aspergillus oryzae and Saccharomyces cerevisiae, are used for sake brewing, leading to highly efficient ethanol fermentation. The final ethanol concentration is $\sim 20 \%$, which is higher than that of beer and wine. The use of technology in the sake brewing process is remarkably high and involves parallel double fermentation. In contrast, beer is brewed using malt to convert the starch in barley into sugar, which is then converted into ethanol by beer yeast. In winemaking, wine yeast converts sugar from grapes into ethanol. Beer and wine processes can include also serial yeast-based double fermentation. It is well-known that bacteria, mainly lactic acid bacteria, can have a role in the production process of beer (sour beer) and wine (malolactic fermentation) (Berbegal et al., 2019; De Roos et al., 2020; Dysvik et al., 2020; Virdis et al., 2020). On the opposite, several alcoholic beverages, such as sake, are considered fermented only by eukaryotic microorganisms.

Beer, sake, and wine originated long before the discovery of microorganisms. Thus, these alcoholic drinks were produced without understanding the mechanism of ethanol fermentation. Surprisingly, sake undergoes pasteurization, called hiire, in the process of sake storage, and it has been recorded as performed 300 years before Pasteur reported the pasteurization method. The high level of biotechnology involved in sake brewing has been maintained for a long time. Sake breweries are widely distributed in Japan, and there may be much more to learn from the sake brewing process. Sake has different grades depending on the degree of polishing of rice. The highest grade (Daiginjo) is produced using polished rice with $50 \%$ or more removal of the outer layer of the grain. In addition, sake is distinguished by the addition of distilled alcohol. Sake without distilled alcohol is called Junmaishu. This opinion paper proposes Sake as a model matrix to highlight bacterial role in traditional beverages that are considered exclusively mold/yeast-based. In addition, the opinion underlines the importance of considering all microbial determinants for a complete safety assessment.

\section{BACTERIAL DNA IN SAKE}

This article was submitted to

Food Microbiology,

a section of the journal

Frontiers in Microbiology

Received: 03 September 2020

Accepted: 27 January 2021

Published: 04 March 2021

Citation:

Nishida H (2021) Sake Brewing and Bacteria Inhabiting Sake Breweries.

Front. Microbiol. 12:602380. doi: $10.3389 /$ fmicb.2021.602380

Numerous microorganisms are present at the beginning of the sake production process because sake brewing is not performed under completely sterile conditions. The ethanol concentration increases with the growth of sake yeast. Although all (or most) microorganisms die due to the high ethanol concentration, their DNA is present in the final sake product. Thus, the DNA sequences in sake can be analyzed to determine and compare the microbial flora contained in sake (Bokulich et al., 2014; Koyanagi et al., 2016; Terasaki et al., 2017; Tsuji et al., 2018).

The bacterial DNA composition of sake is entirely different from that of the water used in sake production (Terasaki et al., 2018). That is, the bacterial DNA detected in sake is not derived from the water but rather from bacteria that have entered and probably briefly grown during the sake production process. Some lactic acid bacteria can survive at ethanol concentrations of $20 \%$, and 
they cause spoiling when they contaminate sake (Suzuki et al., 2008). We have detected a high rate of lactic acid bacteria DNA sequences in spoiled sakes. Thus, DNA sequence comparison can be used as a powerful tool to analyze bacterial flora composition in sake. However, we must bear in mind that the detection of bacterial DNA in sake does not necessarily indicate that these bacteria are still alive.

\section{BACTERIAL FLORA IN MOTO}

Different sakes generally comprise different chemical components (Akaike et al., 2020), and the diversity of chemical components in sake is lower than that of the bacterial flora (Akaike et al., 2020; Terasaki and Nishida, 2020). Specific bacteria appear to affect specific chemical components during the production of the fermentation starter moto. For example, the ornithine composition increases and the arginine composition decreases during the production process of the fermentation starter yamahai-moto (also known as kimoto; traditional fermentation starter), and ornithine-producing Latilactobacillus sakei has been isolated from yamahai-moto (Tsuji et al., 2018). The bacteriocins produced by Lactococcus lactis inhibit the growth of sake-spoiling bacteria (Taniguchi et al., 2010). Although Bacillus sp. and Staphylococcus sp. predominate the early stage of kimoto production, lactobacilli predominate the late stage (Bokulich et al., 2014). Additionally, bacterial flora composition differs between the fermentation starters yamahai-moto and sokujo-moto (Terasaki et al., 2017).

These findings suggest that bacterial flora in sake may influence the chemical components and quality of sake products. Thus, bacteria that have inhabited a sake brewery for a long time could enter the sake brewing process and may be related to the bacterial flora and the characteristics of that sake.

\section{BACTERIAL ISOLATION FROM HATSUZOE}

The fermentation starter moto is mixed with koji, steamed rice, and water, and this is generally a three-step process, including Hatsuzoe (the first step), Nakazoe (the second step), and Tomezoe (the third step). Because bacteria cannot be isolated from sake products, we isolated bacteria from samples of the first mixture (Hatsuzoe). There are many live bacteria in Hatsuzoe (the first mixture of moto and koji) that can be identified and classified based on their $16 \mathrm{~S}$ rDNA sequences. We obtained 46 isolates from six Hatsuzoe (the first mixture of moto and koji) samples from Brewery Toyama 1 (Terasaki and Nishida, 2020). Of them, $23,12,6,2,2$, and 1 belonged to the bacterial genera Kocuria, Staphylococcus, Bacillus, Leifsonia, Microbacterium, and Enterococcus, respectively (Terasaki and Nishida, 2020). The detection of DNA associable to bacteria belonging to these genera underlines the importance, among the future perspectives, of a complete microbial safety assessment in sake productions that includes also prokaryotic organisms. In fact, some of these taxonomic units encompass species/strains of interest for food fermentations, but also undesired species/strains in the food chains (e.g., Tamang et al., 2017; Hanchi et al., 2018; Wu et al., 2019).

We isolated Kocuria sp. from all six Hatsuzoe (the first mixture of moto and koji) samples from Brewery Toyama 1 (Terasaki and Nishida, 2020). We also analyzed 16S rDNA sequencing results from 44 clear sake samples, three cloudy sake samples, and 11 sake-kasu (sake lee) samples from 33 sake breweries. Kocuria DNA was only detected in two samples of cloudy sake and one of sake-kasu, all of which were from Brewery Toyama 1 (Terasaki and Nishida, 2020). Interestingly, Kocuria DNA was not detected in any clear sakes. Our findings indicate that Kocuria DNA was not present in the sake solution because Kocuria cells are difficult to lyse by ethanol (Fujita et al., 2006). Thus, the Kocuria isolates inhabit Brewery Toyama 1 (Terasaki and Nishida, 2020) and are kuratsuki (= living in a sake brewery) bacteria from the sake brewery.

\section{KURATSUKI BACTERIA AND YEAST}

In the past, different sake breweries used different yeast strains that inhabited their brewery, known as kuratsuki yeast. Today, sake brewing using kuratsuki yeast is rare. Many sake breweries buy and use selected sake yeasts that are managed by the Brewery Society of Japan (Jozo-kyokai). However, these yeasts were once established in selected sake breweries.

To our knowledge, no kuratsuki bacteria other than lactic acid bacteria had been reported prior to the publication of our paper (Terasaki and Nishida, 2020). Because it was thought that bacteria do not have a positive effect on the sake brewing process and cause negative effects, such as spoilage, kuratsuki bacteria have not been investigated. All or most kuratsuki yeasts are different strains of Saccharomyces cerevisiae. On the other hand, kuratsuki bacteria may differ at the genus or species level because Kocuria DNA was not detected in any other sake breweries except for Brewery Toyama 1 (Terasaki and Nishida, 2020). Thus, we hypothesize that kuratsuki bacteria have different functions in the sake production process in different sake breweries. We have sequenced the genomic DNA of the Kocuria isolates and are currently analyzing them. We believe that kuratsuki bacteria affect the sake quality, resulting in tastes and flavors that are specific to the brewery producing the sake.

Staphylococcus sp. and Bacillus sp. were isolated from 4/6 and 3/6 Hatsuzoe (the first mixture of moto and koji) samples, respectively, from Brewery Toyama 1 (Terasaki and Nishida, 2020), suggesting that these isolates may also be kuratsuki bacteria. Furthermore, Staphylococcus sp. and Bacillus sp. DNA were detected more frequently in sake than Kocuria DNA (Terasaki and Nishida, 2020). Thus, these DNAs are not specific to bacterial DNA in the sakes of Brewery Toyama 1 (Terasaki and Nishida, 2020) at the genus level. However, if the Staphylococcus sp. and Bacillus sp. isolates are specific to the sake brewery, their genomic sequences could differ at the species or strain level in each genus. 


\section{FUTURE PLAN}

The hiire pasteurization process halts the activities of enzymes of koji mold and sake yeast during the sake production process. Although enzymes, such as amylase and alcohol dehydrogenase, are essential for sake brewing, sake cannot be produced using only rice and these enzymes. Microorganisms are essential for sake production because they produce flavor and taste and influence the sake quality. Although bacteria die at the final stage of sake production, recent studies have shown that bacteria in sake are alive and grow temporarily during the sake production process. Thus, in the production process of sake, a bacterium interacts with another bacterium and sake yeast. Kuratsuki bacteria may play a key role in the interaction among microorganisms and may influence the sake quality. We are studying the functions of kuratsuki bacteria during sake brewing using comparative genome analyses and co-culturing experiments with these bacteria and sake yeast. We plan to change the characteristics of sake and produce a unique sake with different tastes and flavors by exchanging the kuratsuki bacteria

\section{REFERENCES}

Akaike, M., Miyagawa, H., Kimura, Y., Terasaki, M., Kusaba, Y., Kitagaki, H., et al. (2020). Chemical and bacterial components in sake and sake production process. Curr. Microbiol. 77, 632-637. doi: 10.1007/s00284-019-01718-4

Berbegal, C., Borruso, L., Fragasso, M., Tufariello, M., Russo, P., Brusetti, L., et al. (2019). A metagenomic-based approach for the characterization of bacterial diversity associated with spontaneous malolactic fermentations in wine. Int. J. Mol. Sci. 20:3980. doi: 10.3390/ijms20163980

Bokulich, N. A., Ohta, M., Lee, M., and Mills, D. A. (2014). Indigenous bacteria and fungi drive traditional kimoto sake fermentations. Appl. Environ. Microbiol. 80, 5522-5529. doi: 10.1128/AEM.00663-14

Capozzi, V., Fragasso, M., and Russo, P. (2020). Microbiological safety and the management of microbial resources in artisanal foods and beverages: the need for a transdisciplinary assessment to conciliate actual trends and risks avoidance. Microorganisms 8:306. doi: 10.3390/microorganisms8020306

Capozzi, V., Russo, P., and Spano, G. (2012). Microbial information regime in EU geographical indications. World Pat. Inf. 34, 229-231. doi: 10.1016/j.wpi.2012.04.001

Capozzi, V., and Spano, G. (2011). Food microbial biodiversity and "microbes of protected origin”. Front. Microbiol. 2:237. doi: 10.3389/fmicb.2011.00237

De Roos, J., Verce, M., Weckx, S., and De Vuyst, L. (2020). Temporal shotgun metagenomics revealed the potential metabolic capabilities of specific microorganisms during lambic beer production. Front. Microbiol. 11:1692. doi: $10.3389 /$ fmicb.2020.01692

Dysvik, A., La Rosa, S. L., Liland, K. H., Myhrer, K. S., Østlie, H. M., De Rouck, G., et al. (2020). Co-fermentation involving Saccharomyces cerevisiae and Lactobacillus species tolerant to brewing-related stress factors for controlled and rapid production of sour beer. Front. Microbiol. 11:279. doi: $10.3389 /$ fmicb.2020.00279

Fujita, K., Hagishita, T., Kurita, S., Kawakura, Y., Kobayashi, Y., Matsuyama, A., et al. (2006). The cell structural properties of Kocuria rhizophila for aliphatic alcohol exposure. Enzyme Microbial Technol. 39, 511-518. doi: 10.1016/j.enzmictec.2006.01.033

Hanchi, H., Mottawea, W., Sebei, K., and Hammami, R. (2018). The genus Enterococcus: between probiotic potential and safety concerns-an update. Front. Microbiol. 9:1791. doi: 10.3389/fmicb.2018.01791

Koyanagi, T., Nakagawa, A., Kiyohara, M., Matsui, H., Tsuji, A., Barla, F., et al. (2016). Tracing microbiota changes in yamahasi-moto, the traditional Japanese sake starter. Biosci. Biotechnol. Biochem. 80, 399-406. doi: 10.1080/09168451.2015.1095067 from different sake breweries during the production process. Interest in sake is increasing around the world. Some Sake products are Geographical Indications (GIs) (https://www.nta. go.jp/publication/pamph/sake/04.pdf). In effect, an increasing interest is recognized to autochthonous microorganisms in the production of fermented traditional products (Capozzi and Spano, 2011; Capozzi et al., 2012, 2020). This opinion paper claims a possible role for autochthonous bacteria isolated from the sake productions among the factors included in the product specifications. At the same time, it highlights the importance of a complete microbial safety assessment that involves also bacteria.

\section{AUTHOR CONTRIBUTIONS}

HN wrote the manuscript.

\section{FUNDING}

This study was supported by a fund of University collaboration acceleration project of Toyama Prefecture.

Suzuki, K., Asano, S., Iijima, K., and Kitamoto, K. (2008). Sake and beer spoilage lactic acid bacteria-a review. J. Inst. Brew. 114, 209-223. doi: 10.1002/j.2050-0416.2008.tb00331.x

Tamang, J. P., Holzapfel, W. H., Shin, D. H., and Felis, G. E. (2017). Editorial: Microbiology of ethnic fermented foods and alcoholic beverages of the world. Front. Microbiol. 8:1377. doi: 10.3389/fmicb.2017.01377

Taniguchi, M., Ishiyama, Y., Takata, T., Nakanishi, T., Kaneoke, M., Watanabe, K., et al. (2010). Growth-inhibition of hiochi bacteria in namazake (raw sake) by bacteriocins from lactic acid bacteria. J. Biosci. Bioeng. 109, 570-575. doi: 10.1016/j.jbiosc.2009.11.015

Terasaki, M., Fukuyama, A., Takahashi, Y., Yamada, M., and Nishida, H. (2017). Bacterial DNA detected in Japanese rice wines and the fermentation starters. Curr. Microbiol. 74, 1432-1437. doi: 10.1007/s00284-017-1337-4

Terasaki, M., Miyagawa, S., Yamada, M., and Nishida, H. (2018). Detection of bacterial DNA during the process of sake production using sokujo-moto. Curr. Microbiol. 75, 874-879. doi: 10.1007/s00284-018-1460-x

Terasaki, M., and Nishida, H. (2020). Bacterial DNA diversity among clear and cloudy sakes, and sake-kasu. Open Bioinf. J. 13, 74-82. doi: 10.2174/1875036202013010074

Tsuji, A., Kozawa, M., Tokuda, K., Enomoto, T., and Koyanagi, T. (2018). Robust domination of Lactobacillus sakei in microbiota during traditional Japanese sake starter yamahai-moto fermentation and the accompanying changes in metabolites. Curr. Microbiol. 75, 1498-1505. doi: 10.1007/s00284-018-1551-8

Virdis, C., Sumby, K., Bartowsky, E., and Jiranek, V. (2020). Lactic acid bacteria in wine: technological advances and evaluation of their functional role. Front. Microbiol. doi: 10.3389/fmicb.2020.612118

Wu, S., Huang, J., Zhang, F., Wu, Q., Zhang, J., Pang, R., et al. (2019). Prevalence and characterization of food-related methicillin-resistant Staphylococcus aureus (MRSA) in China. Front. Microbiol. 10:304. doi: 10.3389/fmicb.2019.0 0304

Conflict of Interest: The author declares that the research was conducted in the absence of any commercial or financial relationships that could be construed as a potential conflict of interest.

Copyright (c) 2021 Nishida. This is an open-access article distributed under the terms of the Creative Commons Attribution License (CC BY). The use, distribution or reproduction in other forums is permitted, provided the original author(s) and the copyright owner(s) are credited and that the original publication in this journal is cited, in accordance with accepted academic practice. No use, distribution or reproduction is permitted which does not comply with these terms. 